\title{
Medical Image(s) Watermarking and its Optimization using Genetic Programming
}

\author{
Rafi Ullah Habib ${ }^{1}$, Hani Ali Alquhayz ${ }^{2}$ \\ Department of Computer Science and Information \\ College of Science, Majmaah University, Majmaah 11952, Saudi Arabia
}

\begin{abstract}
In this paper, an medical image watermarking technique has been proposed, where intelligence has been incorporated into the encoding and decoding structure. The motion vectors of the medical image sequence are used for embedding the watermark. Instead of a manual selection of the candidate motion vectors, a generalized approach is used to select the most suitable motion vectors for embedding the watermark. Genetic programming (GP) module has been employed to develop a function in accordance with imperceptibility and watermarking capacity. Employment of intelligence in the system improves its imperceptibility, capacity, and resistance toward different attacks that can occur during communication and storing. The motion vectors are generated by applying a blockbased motion estimation algorithm. In this work, Full-Search method has been used for its better performance as compared to the other methods. Experimental results show marked improvement in capacity and visual similarity as compared to the conventional approaches.
\end{abstract}

Keywords-Capacity; imperceptibility; genetic programming; image sequence; watermarking

\section{INTRODUCTION}

The exponential growth of internet speed wherewith the information is made available turned the internet into a place. About a third of the total world population has internet access [1], and consequently, with this development, the spread of multimedia data significantly aroused. Copying, distribution, and manipulation of digital content are very easy by using advanced multimedia and communication tools. Thus, before storing and transmission of digital content, its security is a major concern. Authentication of multimedia applications, especially for sensitive data likes medical and military applications is the challenge of today's research.

For the security of digital content, watermarking is one of the best solutions. There are three main contradictory watermarking properties: robustness, capacity, and imperceptibility [2]. The researchers must make a tradeoff between these properties accordingly. Imperceptibility, robustness, security, and efficient technique(s) are the basic requirements while securing digital data [3]. Watermarking is the well-established solution to many problems like copyright protection, copy control, authentication, ownership, broadcast monitoring etc. [4-6]. In this paper, the secure transmission and storage of medical image sequence like Ultrasound images are ensured by employing the generalized watermarking algorithm using genetic programming. The capacity and imperceptibility are the contradicting parameters and it is difficult to improve any of the parameters without affecting the other one.
However, in this paper, an intelligent approach is employed, where the optimal motion vectors are selected for watermark embedding. Before embedding the watermark bit, the motion vectors are extracted. For this purpose, several block-based motion estimation algorithms are used. Some of them are FullSearch Block-Based Motion-estimation Algorithm (BMA), three-step search, new three-step search, four-step search, diamond search, small diamond search pattern etc. In the proposed technique, Full-Search BMA is used for extracting the motion vectors. Full-Search is more efficient in terms of accuracy as compared to all other BMAs [7]. The computational time of Full-Search BMA is high, but its accuracy is better than all other BMAs. Full-Search BMA searches all of the probabilistic similarity. The magnitude and phase angle between the consecutive motion vectors have been used for watermark embedding. It is not necessary that the motion vectors having large magnitude are suitable for watermark embedding. It depends on the associated macroblock. We cannot rely directly on the magnitude of the vector and thus, we will focus on the associated macroblock parallelly. GP is used to select the most suitable motion vectors for embedding purpose, where imperceptibility, security, and embedding capacity are focused.

The storing and distant transmission of medical images/sequences demands high security and especially medical imaging with mobile transmission needs careful handling. Thus, in this paper, a blind generalized semi-fragile watermarking scheme is used to protect the medical image and/or image sequence. The primary objectives of this paper are:

- Unlike traditional blocked-based security, efficient micro-level security is achieved by applying the FullSearch BMA.

- The temporal information of the input medical image sequence is used for watermark embedding. The embedding capacity is increased as compared to the convention embedding in spatial regions of the images.

- Enhancing the important contradicting properties i.e. imperceptibility, robustness, and capacity is a challenge for today's researchers. In this paper, an efficient tradeoff between these contradicting properties has been made. For this purpose, these parameters are used in the GP fitness function. Details about the GP fitness function are given in Section 3. 
- For imperceptibility, peak signal to noise ratio (PSNR) and structural similarity index measure (SSIM) are used. Determining the capacity of the watermark is to find how much watermark bits are embedded in the image/video without any perceptual distortion [8]. For watermarking capacity (WC), a number of embedded bits per frame (EBPF) are considered and this measurement is used in the fitness function. By the end of all frames embedding, an average of the embedded bits per frame is taken.

\section{LITERATURE SURVEY}

Since the last three decades, the researchers are working on the security of medical images. Most of the researchers are using watermarking methods based on error correcting code, multiple watermarking, hybrid watermarking, intelligent watermarking, biometric watermarking, joint compression based watermarking, etc. Some of the proposed techniques are discussed as follow.

In [9], the motion vector watermarking was proposed where the authors have used the motion vectors having large values and less change in phase angle. The experimental results in their work indicate that the visual similarity of the watermarked image is not highly affected. The MPEG compression speed is not affected and has the capability to embed the high capacity watermark in a short video.

Medical image watermarking technique has been proposed in Singh et al. [10], in which the image is decomposed by using discrete wavelet transform (DWT) and the medical text watermark is embedded in the sub-bands of the wavelet domain using spread spectrum technique. Third level decomposition is made and based on threshold criteria, three different watermarks have been embedded in the selected vertical and horizontal wavelet subbands.

In [11], Giakoumaki et al. have proposed wavelet-based watermarking algorithms, where the authors address the issues related to medical confidentiality. The input image is decomposed by using wavelet transform and the watermark is embedded in the wavelet coefficients. The authors have utilized two watermarking approaches for medical images. The detailed coefficients of the cover image are used for watermark embedding. Quantization function has been applied to each of the marked coefficients for extracting the multiple watermark bits. By using this hybrid algorithm, robustness, reliability, efficiency, and imperceptibility have been improved. The only disadvantage of this method is higher computational complexity.

In [12], Ouhsain et al. proposed a discrete fractional Fourier and DWT based watermarking system. After first level wavelet decomposition, the input image is divided into four subbands. Multiple parameters discrete fractional Fourier (MPDFRF) is applied to each subband and the watermark is embedded into each block.

A blind optimal watermarking method has been proposed by Peng et al. [13]. This method is based on the multi-wavelet transformation and support vector machine (SVM). After first level wavelet decomposition, the lower frequency sub-band of the cover image is used for watermark embedding. The reference information and logo have been used as a watermark to be embedded.

A biometric-based medical image watermarking method has been proposed by Wioletta [14]. The author is using DWT to embed a biometric watermark into the cover work. This method is robust against both legitimate and illegitimate manipulations.

Lin and Ching proposed a blind wavelet-based digital image watermarking scheme that has high capacity and able to hide more than one image inside the cover work without affecting the imperceptibility of the watermarked image [15]. The watermark is permuted before embedding to ensure the robustness and security of the watermark simultaneously.

In 2009, I. Usman et al., have proposed an intelligent watermarking technique [16]. The authors intelligently select the coefficient for watermark embedding. The embedding coefficients are selected using genetic programming. The authors are exploiting the amplitude of the wavelet coefficient. GP has been employed for evolving the mathematical function, which is based on visual similarity and watermarking capacity. The results are effective in terms of imperceptibility and payload.

S. Acharjee et al. have proposed a watermarking technique that enhances the security of medical videos [17]. Motion vectors of the electrocardiogram (ECG) videos are used for watermark embedding. A binary image is used as a watermark. The authors are using a threshold to select the motion vectors. In addition, they have tried to reduce the calculation and speed up the authentication process.

\section{Genetic Programming (GP) Module}

Genetic programming is an evolutionary computation technique that is used in many applications. GP is a machine learning technique based on natural selection and genetics [18]. GP is based on the stochastic method, where randomness plays an important role in searching and learning [19]. The most suitable motion vectors, in terms of visual similarity and capacity, are selected for embedding watermark bits. Initially, a random population is evaluated using a fitness function according to the application. The individuals in the population are computer programs. In the proposed scheme, the fitness function that is based on imperceptibility and capacity is focused. In the initial population, the best individual is reserved and all of the others are deleted and replaced by the children of the best individuals. The reserved children make a new generation, where so of the children may have the best score than their parents in the previous generation. This process is repeated until one of the termination criterions is satisfied. Block diagram for developing GP module is shown in Fig. 1.

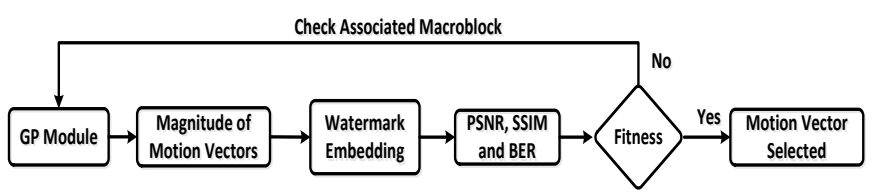

Fig. 1. Basic GP Architecture. 
Fitness criteria, suitable functions, and terminals are defined that represent the possible solutions in the form of a complex numerical function. GP module has the following different functions.

\section{A. GP Function Set}

The function set used in GP is based in the nature $f$ the problem. The available mathematical function in GP module collectively makes the GP function set. In the simulations, four basic binary functions have been,,$+- \times, \div, \log$, exponent are used.

\section{B. Fitness Function}

Each individual of the population is graded. In the proposed scheme, the PSNR, SSIM, and capacity are used to assess the fitness function. This function provides the feedback to the GP module representing the fitness of the individual. A higher individual fitness score indicates higher performance. GP fitness function contains four basic arithmetic operators and the operands like magnitude and phase angle of motion vectors, macroblock index. The performance evaluation of the fitness function is measured by the PSNR, SSIM and watermarking capacity. The following fitness function is used:

fitness $=\alpha_{1} \times \frac{\text { PSNR }}{50}+\alpha_{2} \times \operatorname{SSIM}+\alpha_{3} \times \mathrm{WC}$

The watermarking capacity is $\mathrm{WC}=\mathrm{EBPF} /(\mathrm{M} \times \mathrm{N})$, where $\mathrm{M} \times \mathrm{N}$ is the size of the frame. The constants $\alpha_{1}, \alpha_{2}$ and $\alpha_{3}$ are the weighting parameters that are decided according to the application. For applications requiring more capacity/payload, some sacrifice can be made in terms of imperceptibility, i.e. the parameter $\alpha_{3}$ should be given more weight. Similarly, for the applications, where high imperceptibility is required, the parameters $\alpha_{1}$ and $\alpha_{2}$ should be given more weight. PSNR and SSIM are calculated between the original image and the watermarked image. PSNR is divided by 50 in order to scale its value, such that equal weight could be given to all of the objective measures.

\section{Population Initialization}

The individuals in the initial population are generated randomly. The most common methods that are used for the initialization of population are "ramped half - and - half" and "full - and - grow" methods. By using any of the abovementioned methods, the pre-defined maximum tree depth does not be exceeded [20]. In the proposed scheme, the "Ramped half - and - half" method is used for generating the initial population.

\section{Termination Criteria}

The simulation is terminated when one of the following becomes true.

- When the fitness score is exceeded i.e. fitness $>50$

- When the fitness score repeats

- When the number of generations reaches the predefined maximum number of generations

- When the error becomes less than a pre-defined threshold

\section{E. GP Operators}

The most common operators used in genetic programming are crossover, replication and mutation. In the proposed scheme, crossover, mutation and replication are used to produce the next generation. In a crossover, the two individual parents exchange genetic materials to create a child. It tries to mimic recombination and reproduction. Optimal and/or near to optimal solution comes with the help of crossover operator. In mutation, the genome is changed in a minor way for the next generation. In replication, the individual is copied to the new generation. In the proposed GP run, a crossover has a higher ratio as compared to the mutation and replication. All of the necessary settings of the GP module are given in Table I.

TABLE I. GP PARAMETERS SETTINGS

\begin{tabular}{|l|l|}
\hline Objectives & To evolve the best result \\
\hline Function Operators & Log, Exponent,,,$+- \times, \div$ \\
\hline Function Operands & $\begin{array}{l}\text { The magnitude and the Phase angle of motion } \\
\text { vectors }\end{array}$ \\
\hline Fitness & PSNR, SSIM and watermarking capacity \\
\hline Expected children & rank89 \\
\hline Selection & General \\
\hline Initial Population & ramped half - and - half \\
\hline Termination & Score exceed, Number of generations, error \\
\hline Sampling & Tournament \\
\hline Survival & Keep the best \\
\hline GP Operators & Mutation, Crossover, Replication \\
\hline
\end{tabular}

\section{PROPOSED AlgORITHM}

A generalized watermarking algorithm for the ultrasound image sequence has been presented. The temporal space is acquired by using a Full Search block-based motion estimation algorithm. As compared to all other block-based motion estimation algorithms, Full Search is time-consuming but it is more efficient. The intelligent selection is carried out while choosing the candidate motion vectors. For this purpose, genetic programming is used to select the efficient motion vectors where the watermark bits are embedded. This will increase the visual similarity, capacity and the robustness of the watermarking system.

MATLAB environment has been used for the experimental results. For GP simulation, the Matlab based GPLAB toolbox has been used [21].

\section{A. Watermark Embedding}

- A key-based random binary sequence is generated that is used as a watermark in the proposed system. The watermark is quadruplicated and then scrambled before embedding in the motion vectors. $\mathrm{b}=\operatorname{rand}(\mathrm{i})$, where $\mathrm{i}$ is the number of watermark bits quarter to the required bits. The randomly generated bits are then quadruplicated to make a vector $\mathrm{V}$ i.e. $\mathrm{V}=$ $\left(b_{1}, b_{2}, \ldots b_{n}, b_{1}, b_{2}, s \ldots b_{n}, b_{1}, b_{2}, \ldots b_{n}, b_{1}, b_{2}, \ldots b_{n}\right)$ where $\mathrm{n}$ is the number of generated bits. The quadruplicated bits are then permuted by using a secret 
key to make it more secure [22]. Thus the final watermark to be embedded is: $\mathrm{W}_{\text {final }}=\mathrm{V}_{\text {permuted }}$.

- We are applying a full-search block-based motion estimation algorithm to the ultrasound image sequence to generate motion vectors.

- The genetic programming module (GP) is introduced, where the initial population, generations and fitness function, termination criteria etc. are decided. In this paper, the fitness function is based on the PSNR and SSIM along with the watermarking capacity.

- For embedding the watermark, the most suitable candidate motion vectors are selected by using the above-mentioned genetic programming module.

- The watermark bits are embedded in the motion vectors and run generation by generation until the termination criteria achieved. The termination criteria can fitness score, a number of generation, execution time or repetition of the fitness values. The numerical fitness expressions are evaluated while embedding the watermark bits in the ultrasound frames. These expressions are used in the watermark extraction. This is to be discussed in detail in Section 4.2.

- Once the motion vector is selected, its magnitude and the phase angle are modified. The magnitude of the motion vector is given in Equation 2.

$\operatorname{Mag}_{m v}(i)=\sqrt{h_{i}^{2}+v_{i}^{2}}, \quad 0<i \leq M B$

Where $\mathrm{MB}$ is the macroblock, $\mathrm{h}$ and $\mathrm{v}$ are the horizontal and vertical values of the motion vector respectively. Calculate the phase angle to embed the watermark bit. The phase angle is given in Equation 3.

$$
\theta(\mathrm{j})=\tan ^{-1} m v(v)_{i} / m v(h)_{i}
$$

where $\operatorname{mv}(\mathrm{v})$ and $\operatorname{mv}(\mathrm{h})$ are the vertical and horizontal components respectively.

- If the watermark bit is 1 then we chose the coordinate sectors I and II, otherwise, we chose the coordinate sectors III and IV for embedding. If the $0 \leq \theta \leq 90$, then modify the horizontal component of the selected vector and if $90<\theta \leq 180$ then the vertical component of the motion vectors is modified [23]. The coordinate regions are shown in Fig. 2.

The effect of watermark embedding is measured by PSNR and SSIM as given in Equation 4 and Equation 5.

$P S N R=20 \log _{10}\left(\frac{255^{2}}{\mathrm{MSE}}\right)$

where MSE is the mean square error.

$$
\operatorname{SSIM}(x, y)=\frac{\left(2 \mu_{x} \mu_{y}+c_{1}\right)\left(2 \sigma_{x y}+c_{2}\right)}{\left(\mu_{x}^{2}+\mu_{y}^{2}+c_{1}\right)\left(\sigma_{x}^{2}+\sigma_{y}^{2}+c_{2}\right)}
$$

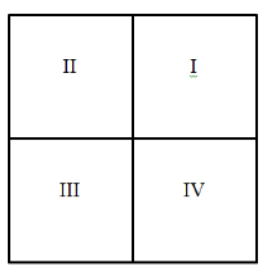

Fig. 2. The Coordinate System.

where $\mathrm{x}$ and $\mathrm{y}$ are two windows of size $\mathrm{N} \times \mathrm{N}, \mu_{\mathrm{x}}$ and $\mu_{\mathrm{y}}$ are the averages of $\mathrm{x}$ and $\mathrm{y}$, and $\sigma_{\mathrm{x}}^{2}$ and $\sigma_{\mathrm{y}}^{2}$ are the variances of $\mathrm{x}$ and $\mathrm{y}$ respectively. $\mathrm{c}_{1}=\left(\mathrm{K}_{1} \mathrm{~L}\right)^{2}$ and $\mathrm{c}_{2}=\left(\mathrm{K}_{2} \mathrm{~L}\right)^{2}$ where $\mathrm{L}$ is the maximum value i.e. $2^{\# \text { bites }}$ and $\mathrm{k}_{1}=0.01$ and $\mathrm{k}_{2}=0.03$ by default.

Normally, the watermark capacity is calculated as bits per pixel (bpp), when the spatial domain is used for watermark embedding. However, in the proposed scheme, the capacity is calculated by the embedded bits in the frame.

When the fitness criteria, which are PSNR, SSIM and capacity are fulfilled, the system will move on to the next frame for watermark embedding and continue until embedding all the frames of the sequence.

\section{B. Watermark Extraction}

Once the ultrasound image sequence watermarking is finished and shared through a wired or wireless communication channel, then verification is required on the receiving side.

- On the receiving side, motion vectors of the watermarked image sequence are generated. Then we select those motion vectors, which were used for watermark embedding. For a selection of the marked candidate motion vectors, the fitness numerical expression is used. The same fitness function equation is used to select the motion vectors.

- The watermark bits are then extracted from the selected motion vectors and compared to the same watermark bits that can be generated on the verification side by using the same key.

- If the extracted watermark bits and the generated watermark bits are same, then the image sequence is authentic, otherwise tampered.

The watermark embedding and extraction are shown in Fig. 3 and 4, respectively.

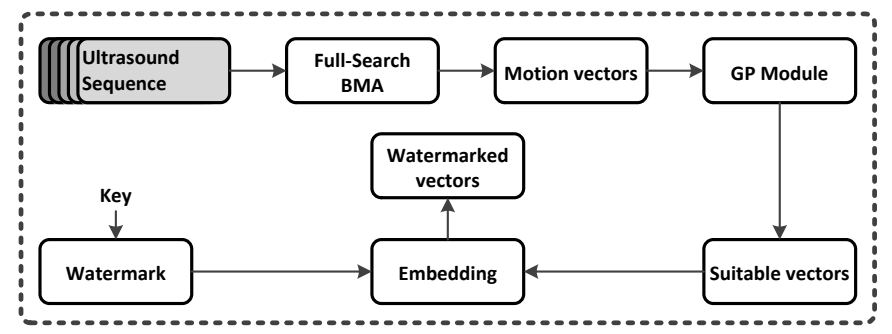

Fig. 3. Watermark Embedding Process. 


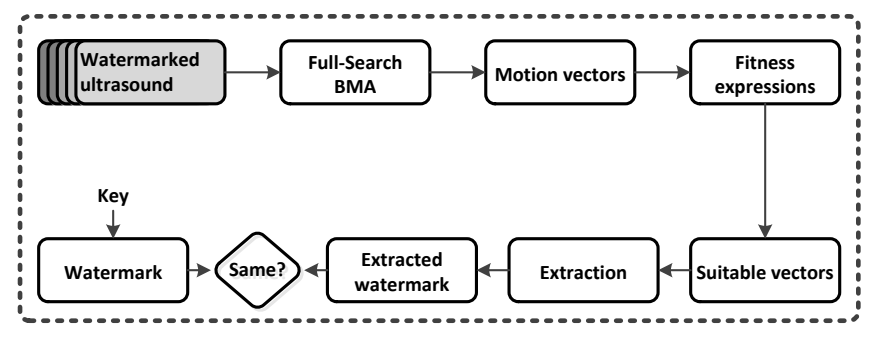

Fig. 4. Watermark Extraction Process.

In the extraction process, the to-be-checked-sequence is used as an input and the motion vectors are selected where the watermarks bits were embedded on the embedding side. For the selection of the motion vectors, the fitness function expressions are used that were evaluated by GP module. The watermark is generated by using the same key and then the generated and extracted watermarks are compared. If both the watermarks are same, the sequence is authentic otherwise tampered. The location(s) of tampering is then analyzed and detected that which frame(s) tampers and how much. For normal videos, it is decided that the tampering is acceptable or not. However, for medical videos, a tiny distortion cannot be accepted. For this reason, the legitimate and illegitimate tampering is not differentiated in this paper.

\section{EXPERIMENTAL RESULTS}

An ultrasound sequence has been chosen for the experimental work [24]. After applying the Full-Search BMA, lots of motion vectors are obtained. Thus, a high strength watermark can be embedded without affecting the imperceptibility. Although, it is not necessary that large magnitude vectors are suitable for watermark embedding. It depends on the associated macroblock. We cannot rely directly on the magnitude of the vector and thus, the associated macroblock is focused parallelly. Any fixed threshold is not assigned to the magnitude of the motion vectors for the embedding selection. For this purpose, an intelligent system is employed, where the GP module will decide the most suitable motion vector for embedding the watermark bits. The details of the ultrasound sequence before and after Full-search BMA are given in Table II.

TABLE II. DETAIL OF THE ULTRASOUND IMAGE SEQUENCE BEFORE AND AFTER FULL-SEARCH BMA

\begin{tabular}{|l|l|}
\hline Features & Details \\
\hline Image sequence / video & Ultrasound video \\
\hline Resolution & $1920 \times 1080$ \\
\hline Size & $97 \mathrm{MB}$ \\
\hline Aspect Ratio & $16: 9$ \\
\hline Frame rate & $25.00 \mathrm{fps}$ \\
\hline Duration & $00: 06$ \\
\hline Total frames & 168 \\
\hline BMA & Full-Search \\
\hline Macroblock size & $16 \times 16$ \\
\hline Number of motion vectors/frame & 8100 \\
\hline Motion vector threshold for selection & GP based \\
\hline
\end{tabular}

Fig. 5 and 6 show some of the frames extracted from the video and the corresponding motion vectors respectively. The structures of medical images/videos are much different from normal videos. Hence it is difficult to compare any watermarking algorithm that is designed for normal videos i.e. . bmp, tiff, . jpeg etc.

The watermarked motion vectors are shown in Fig. 7. In Fig. 5 and 6 , it can be seen that there are hundreds of motion vectors even if the size of the macroblock is $16 \times 16$. Hence, the embedding capacity is too high as compared to the regular images. In the proposed method, the motion vectors are intelligently selected for embedding the watermark bits; therefore, the visual similarity can be easily maintained after embedding a high strength watermark. If the motion vectors on the boundary are selected for embedding then it may create some problem of overflow and underflow. To avoid the overflow/underflow, a histogram modification is used before embedding the watermark. The phenomenon used in [25] has been utilized where the authors have proposed a preprocessing method for fixing the issue of overflow and underflow. They find the zero point that is closed to the boundary i.e. (0 or 255), and the boundary pixels are removed and the pixels between the boundary pixels and zero points are removed by modifying the histogram of the original frame. For histogram generating, the boundary pixels are not used and after embedding the watermark, new boundary pixels appear. Thus, a correct histogram can be obtained, while extracting the watermark on the verification side.

It is not easy to compare medical images with normal images like bmp, jpeg, and tiff images. The dicom image contains the details about the image and has patient information in the same file. It contains the header and other information that are structured differently from the other formats. Little Endian or Big Endian byte orders are used to encode the dicom image file format. Dicom formats are converted to bmp because the main interest is the security of the image rather than the patient and machine information associated with the images. The converted images are viewable on the common image viewing system with small size [26].

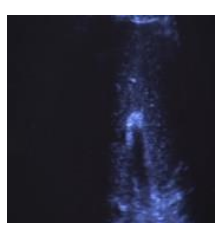

(a)

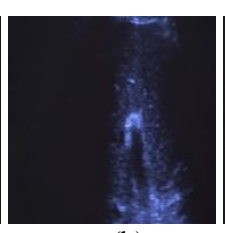

(b)

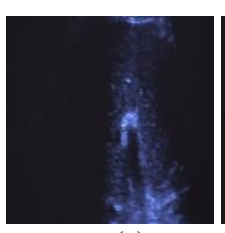

(c)

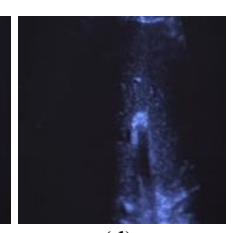

(d)
Fig. 5. From a $\sim$ d, the Frames Number 7, 8, 9 and 10 of the Ultrasound Sequence.

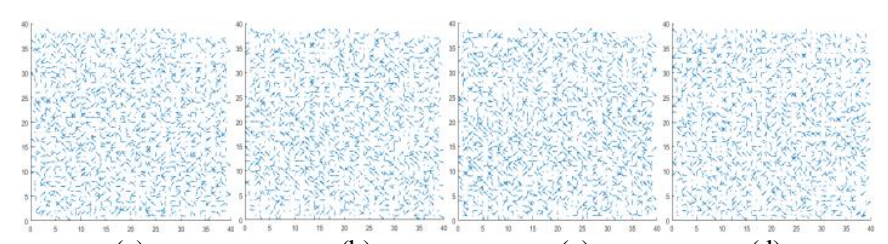

(a) (b)

(c)

(d)

Fig. 6. From a d, the Extracted Motion Vectors. 


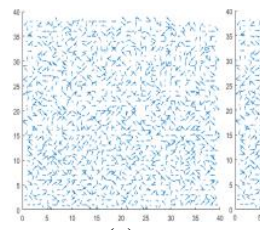

(a)

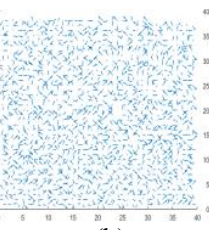

(b)

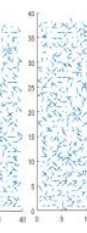

(c) (c)
Fig. 7. From $\mathrm{a} \sim \mathrm{d}$, the Watermarked Motion Vectors.

Table III shows the embedding distortion that is measured by PSNR and SSIM along with the embedding capacity. The main objective of the proposed approach is to increase the embedding capacity with minimum effect on imperceptibility and vice versa. The results of the proposed algorithm have been compared with the three latest schemes. It can be seen that the proposed approach provide an optimal solution to the contradicting properties of watermarking. As compared to other regular video frames, in medical images, there are more motion vectors. In addition, optimal motion vectors are selected for embedding the watermark bits.

In Table III, it can be seen that the PSNR and SSIM in the second column are very high. The SSIM i.e. 0.99 has been calculated for the original and the extracted watermark and it should be one if the watermarked content is not attacked/tampered. Similarly, the SSIM i.e. 0.97 is calculated for the watermarked image and received the watermarked image. All others SSIMs and PSNRs, shown in Table III are used to measure the embedding distortion.

TABLE III. PROMINENT FEATURES AND PERFORMANCE COMPARISON WITH THE PREVIOUS APPROACHES

\begin{tabular}{|l|l|l|l|l|}
\hline Features & {$[\mathbf{1 7}]$} & {$[\mathbf{2 5}]$} & {$[\mathbf{2 7}]$} & Proposed \\
\hline $\begin{array}{l}\text { Embedding } \\
\text { domain }\end{array}$ & Temporal & Spatial & Transformed & Temporal \\
\hline PSNR & $\sim 120 \mathrm{~dB}$ & $\begin{array}{l}\sim 50 \mathrm{~dB}(\text { compressed } \\
\text { watermark })\end{array}$ & $\begin{array}{l}35 \mathrm{~dB}- \\
\begin{array}{l}36 \mathrm{~dB} \\
\text { (Depending } \\
\text { on the gain } \\
\text { factor, } \alpha)\end{array}\end{array}$ & $\begin{array}{l}\sim 43 \mathrm{~dB} \\
\text { (Average) }\end{array}$ \\
\hline SSIM & $\begin{array}{l}0.99 \& \\
0.97\end{array}$ & Not calculated & $\begin{array}{l}\text { Not } \\
\text { calculated }\end{array}$ & $\begin{array}{l}0.95 \\
\text { (Average) }\end{array}$ \\
\hline Capacity & $\begin{array}{l}\text { Not } \\
\text { mentioned }\end{array}$ & $\begin{array}{l}\text { Using Compressed } \\
\text { watermark }\end{array}$ & High & $\begin{array}{l}\text { High, i.e. } \\
\sim 50 \text { bits } \\
\text { for } \\
(16 \times 16) \\
\text { block }\end{array}$ \\
\hline
\end{tabular}

\section{CONCLUSIONS AND FUTURE DiRECTIONS}

An intelligent watermarking in motion vectors is proposed for protecting the medical videos. Medical images/video is very sensitive and small distortion may not be acceptable. Hence, the selection of pixels and motion vectors for embedding the watermark is a challenge for the researchers and hospital management systems. In this paper, a genetic programming module has been designed to select the most suitable motion vectors for watermark embedding that reduced the perceptual distortion and improve the watermarking capacity. Distortion measurements like PSNR and SSIM along with the watermarking capacity have been used to set the fitness function. The watermark bits are embedded using the magnitude and phase angle of the motion vectors. The imperceptibility and the watermarking capacity have been improved. Random permutation and quadruplicating improve the security of the up to satisfactory level. The experimental results show that the proposed watermarking approach is more efficient in terms of visual similarity and capacity.

Cyber-attacks like data injection, stealing patient information, ransomware, image disruption etc. threat the medical images. In future, an intelligent watermarking system can be employed to increase the security of medical images and to protect the information related to the patient. The watermark that is to be used will contain all of the patient information. A highly compressed version of the patient picture can also be used as a watermark.

\section{ACKNOWLEDGEMENT}

The authors would like to thank Deanship of Scientific Research at Majmaah University for supporting this work under Project No. 38/75.

\section{REFERENCES}

[1] Boneh D, Shaw J. "Collusion-secure fingerprinting for digital data" IEEE Transactions on Information Theory, vol. 44(5), pp. 1897-1905, 1998.

[2] Khan A, Mirza A M. "Genetic perceptual shaping: Utilizing cover image and conceivable attack information during watermark embedding. Information Fusion, “Elsevier, vol. 8 (4), pp. 354-365, 2007.

[3] Chamlawi R, Khan A, Idris A. "Wavelet based image authentication and recovery," Journal of Computer Science and Technology, vol. 22 (6), pp. 795- 804, 2007.

[4] Chang C C, Fan Y H, Tai W L. "Four-scanning attack on hierarchical digital watermarking method for image tamper detection and recovery,“ Pattern Recognition, Elsevier, vol. 41, pp. 654-661, 2008.

[5] Shih F Y, Wu Y T. "Robust watermarking and compression for medical images based on genetic algorithms. Information Sciences," Elsevier, vol. 175 , pp. 200-216, 2005.

[6] Suhail M A, Obaidat M S, Ipson S S,Aadoun B. "A comparative study of digital watermarking in JPEG and JPEG2000 environment,“ Information Sciences, Elsevier, vol. 151, pp. 93-105, 2003.

[7] Yaakob R, Aryanfar A, Halim A A, Sulaiman N. "A Comparison of Different Block Matching Algorithms for Motion Estimation," Procedia Technology, Elsevier, vol, 11, pp. 199-205, 2013.

[8] Yaghmaee F, Jamzad M. "Estimating Watermarking Capacity in Gray Scale Images Based on Image Complexity, EURASIP Journal on Advances in Signal Processing, vol. 2010, pp. 1-9, 2010.

[9] Zhang J, Li J, Hang L. "Video Watermark Technique in Motion Vector," In Proceedings Brazilian Symposium on Computer Graphics and Image Processing, pp. 179-182, 2001.

[10] Singh A K, Kumar B, Dave B, Mohan A. "Robust and imperceptible spread-spectrum watermarking for telemedicine applications, “ In Proc. Natl. Acad. Sci., India, vol. 85(2), pp. 295-301, 2015.

[11] Giakoumaki, Pavlopoulos S, Koutsouris D. "A medical image watermarking scheme based on wavelet transform," in Proceedings of 25th Annual International Conference of IEEE-EMBS, San Francisco. 2004, 1541-1544.

[12] Ouhsain M, AbdallahE E, Hamza A B. "An image watermarking scheme based on wavelet and multiple-parameter fractional Fourier transform," in IEEE Int Conf on Signal Processing and Communication, UAE, pp. 1375-1378, 2007.

[13] Peng H, Wang J, Wang W. Image watermarking method in multiwavelet domain based on support vector machines. Journal of Systems and Software, vol. 83, pp. 1470-1477, 2010.

[14] Wioletta W. "Biometric watermarking for medical images-example of Iris code,“ Tech. Trans, vol. 1-M(5), pp. 409-416, 2013. 
[15] Lin C Y, Tai C Y. "A robust image hiding method using wavelet technique," Journal of Information Science and Engineering, vol. 22, pp. 163-174, 2006.

[16] Usman I, Khan A, Ali A, Choi T S. "Reversible watermarking based on intelligent Coefficient selection and integer wavelet transform,“ International Journal of Innovative Computing, Information and Control, vol. 5(12), pp. 1-8, 2009.

[17] Acharjee S, et al. "Watermarking in Motion Vector for Security Enhancement of Medical Videos," Int Conf on Control, Instrumentation. Comm and Computational Tech, pp. 589-594, 2014.

[18] Banzhaf W, Francone F D, Keller R E, Nordin P, "Genetic programming: an introduction: on the automatic evolution of computer programs and its applications, "Morgan Kaufmann Publishers Inc. San Francisco, CA, USA, 1998

[19] Duda R O, Hart P E, Stork D G. "Pattern Classification,“ 2 $2^{\text {nd }}$ Edition, New York: John Wiley \& Sons, Inc, 2001.

[20] Koza J. "Genetic programming: on the programming of computers by means of natural selection," Cambridge, USA: MIT Press, 1992.
[21] Silva S. "GPLAB-MATLAB programming based toolbox for GP simulation,“ 2015.

[22] Piva A, Bartolini F, Caldelli R. "Self-recovery authentication of images in the DWT domain," International Journal of Image and Graphics, vol. 5(1), pp. 149-166, 2005.

[23] Ullah R, Alquhayz H A. "Authentication of Topographic EEG: Employing Transform Based Watermarking," International Journal of Computer Science and Network Security, vol. 17(3), pp. 43-54, 2017.

[24] https://www.videvo.net/video/ultrasound-scan-5/3306/.

[25] Badshah G, Liew S C, Zain J M, Ali M. "Watermarking of ultrasound medical images in teleradiology using compressed watermark," Journal of Medical Imaging, SPIE, vol. 3(1), pp.. 170011-170019, 2016.

[26] Ujgare N S, Baviskar S P. "Conversion of DICOM Image in to JPEG, BMP and PNG Image Format, “Int Journal of Computer Applications, vol. 62(11), pp. 22-26, 2013.

[27] Sing K, Kumar B, Singh G, Mohan A. "Robust and imperceptible hybrid watermarking techniques for medical images," Medical Image Watermarking, Springer, pp. 61-93, 2017. 